\section{INTERPRETATION OF SURGICAL NEUROMONITORING DATA IN CANADA: OUR VIEW}

We read with interest the recent Discussions in Surgery article by Norton and colleagues (DOI: 10.1503/ cjs.013214). We commend the authors for their efforts, as their survey highlights some important issues related to intraoperative neuromonitoring (IOM) in Canada. It is encouraging that there is increasing interest in the field. However, the authors point out the lack of trained IOM personnel to meet the needs of surgeons and the lack of consistent IOM training strategies in Canada.

The training of neuromonitoring skills is a key issue because the major thrust of the survey was the interpretation of results. We would like to offer our view on interpretation, as we see fundamental differences between the clinical neurophysiology laboratory and neuromonitoring in the operating room. As described in the paper by Norton and colleagues, the clinical neurophysiology lab typically includes certified technologists providing support for neurologists. The clinical practitioner furnishes a medical diagnosis usually based on several factors, including electrophysiological examinations. In the challenging environment of the operating room, the goal of the neuromonitorist is not diagnosis, but the evaluation of deviations from a set of baseline neurophysiological tests. It is the neuromonitorist's task to identify any deviations from the baseline data set and establish a timely liaison between relevant clinical professionals, which predominantly includes the surgeon and/or anesthesiologist. In this respect, actions taken to rectify the changes in neuromonitoring data are placed in the hands of the surgeon or anesthesiologist. Ultimately, therefore, clinical decisions are made by physician practitioners in response to timely delivered neurophysiological data.

Perhaps the most interesting result of the survey was the preference of surgeons (cardiac surgeons excepted) for experienced doctorallevel interpreters. However, the question posed in the survey may itself be prone to misinterpretation. Reasonably, most surgeons do not want to observe raw neurophysiological data, but would prefer timely, accurate and relevant reports that they can interpret within the context of the ongoing procedure. Regardless of the messenger, we argue that the ultimate clinical interpretation would still reside with the surgeon (or anesthesiologist).

The authors acknowledge the shortage of doctoral level IOM personnel, but we believe this circumstance is unlikely to improve in view of our fiscally tight health care environment. The most reasonable solution would be to ensure a rigorous training program suitable for technologist-level neuromonitorists. In our centre, a doctoral-level neurophysiologist oversees technologists who are required to complete Certification of Neurointraoperative Monitoring. However, readers should also be aware that steps are currently being taken to confront the deficiency in neuromonitoring training in Canada. In September 2014 the Canadian Association of Neurophysiological Monitoring launched Canada's first formal training stream for intraoperative monitoring through The Michener Institute in Toronto, Ont. This course is web-based, thereby increasing access, and includes a practicum upon completion of the 2-year program.

We applaud Norton and colleagues for contributing to the much needed national dialogue on the direction of neurophysiological monitoring in Canada and look forward to continuing progress in this interesting field.

\section{Marshall Wilkinson, MSc, PhD; Anthony Kaufmann, MSc, MD}

Section of Neurosurgery, Faculty of Medicine, University of Manitoba and Health Sciences Centre, Winnipeg, Man.

DOI: $10.1503 /$ cjs.007815

\section{INTERPRETATION OF SURGICAL NEUROMONITORING DATA IN CANADA: AUTHOR RESPONSE}

We thank Drs. Wilkinson and Kaufmann for their interest in our study ${ }^{1}$ and their considered response to it. We thank them for highlighting the Canadian Association of Neurophysiological Monitoring course through the Michener Institute in Toronto, and point out that a training scheme has been approved by the Alberta College of Medical Diagnostic and Therapeutic Technologists (www.acmdtt.ca) at the University of Alberta Hospital, and is also being used in Saskatoon. We agree that patient safety can be improved through the use of highly skilled, well-trained individuals in the operating room.

We differ from Wilkson and Kaufmann with regard to the issue of interpretation, however. In our view, the IOM situation should not be different from that in the outpatient laboratory. A skilled individual performs the technical aspect of the task and provides an impression of the test. The test itself is interpreted by a clinical practitioner. The patient's physician (most responsible physician; MRP) integrates this interpretation with other clinical signs and knowledge to form a diagnosis and treatment plan. Indeed, many reports from the electroencephalography laboratory (or the radiology department, which also follows this model) end with the statement that "clinical correlation is required." In the intraoperative neuromonitoring (IOM) setting we 